\title{
Modélisation numérique du comportement d'atténuateurs de houles flottants sur un site côtier
}

\author{
Jean Bougis \\ Ingénieur Conseil, 34 Chemin du Moulin - 06650 Opio - Tél 93.77.74.22
}

Alex Degaie

Société Degaie, 97 Route de Bavay-59138 Pont sur Sambre - Tél 27.53.61.10

\section{Résumé}

La présente méthode est fondée sur un modèle d'agitation hybride éléments finis / singularités. Une matrice d'impédance, construite en résolvant les équations intégrales tridimensionnelles dans un domaine enfermant la file d'atténuateurs mobiles (six degrés de liberté par atténuateur), est incorporée dans le modèle d'éléments finis bidimensionnel plan de Berkhoff. La méthode permet également d'utiliser une matrice d'impédance tridimensionnelle expérimentale, ou une matrice d'impédance bidimensionnelle numérique ou expérimentale. Le modèle est utilisable, dès l'avant projet, pour prévoir les effets qu'auront différentes configurations d'atténuateurs sur un site côtier. Il a été développé et validé lors de la mise au point du nouveau concept d'atténuateur de houles ASB-5000 et de l'étude de son implantation pour protéger des sites réels.

\section{Introduction}

Pour différentes raisons (techniques, économiques, écologiques ...), l'utilisation de brise-clapots ou d'atténuateurs de houles pour la protection de sites côtiers contre la houle a tendance à devenir de plus en plus fréquente.

Qu'il s'agisse, de brise-lames, d'atténuateurs de houles, de pontons lourds, ou de tout autre type de brise-clapots, une fois que le système de protection le mieux adapté a été choisi et dimensionné en fonction de son comportement hydromécanique et de ses performances (étude hydrodynamique intrinsèque du dispositif), il faut étudier sa mise en place. Cette étude, qui est naturellement d'autant plus nécessaire et délicate à réaliser que le système anti-houles est plus efficace, doit permettre de répondre aux questions suivantes :

Où et comment les atténuateurs doivent-ils être disposés pour obtenir les effets de protection désirés, sans pour autant géner l'utilisation du plan d'eau, ni son accès? Quelle longueur faut-il donner à la protection? Il faut également vérifier que l'effet obtenu, compte tenu de la bathymétrie et des limites existantes ou à venir (digues, plages ...) est bien celui qui était escompté.

La réponse à ce type de questions relève classiquement de la méthode expérimentale sur modèles réduits physiques. Toutefois, dans le cas de protections 
flottantes cette approche peut être remise en cause pour deux raisons principales, l'une technique qui impose de grandes échelles et l'autre économique:

- Les échelles des modèles de génie côtier étant généralement de l'ordre du 1/100 ème, les performances intrinsèques des atténuateurs ou des brise-lames flottants ne sont pas représentées dans de bonnes conditions de similitude. En particulier, d'une part les efforts dus à la viscosité sont très exagérés sur le modèle (écoulements laminaires) par rapport au prototype (écoulements turbulents) même en outrant la rugosité des carènes, et d'autre part les efforts dus à la tension superficielle de l'interface air-eau peuvent modifier considérablement le comportement hydromécanique d'engins flottants de petites dimensions.

- Le coût d'un modèle physique de génie côtier à grande échelle (1/10 à 1/20) permettant de représenter correctement le comportement des atténuateurs peut se révéler presque aussi important que celui de la réalisation du prototype, et ne pas être compatible avec les objectifs financiers de ce type de projet.

Ces deux raisons rendent désormais indispensable, même s'il n'est pas exclusif, le recours à l'utilisation d'un modèle numérique d'agitation portuaire susceptible de prendre en compte l'action des atténuateurs et brise-clapots flottants.

\section{Problème aux limites}

\subsection{Hypothèses}

\subsubsection{Hvpothèses générales}

Le problème hydrodynamique est résolu dans le cadre de la théorie linéarisée des écoulements irrotationnels à surface libre des fluides parfaits.

L'amplitude et la cambrure des ondes de gravité sont supposées être suffisamment faibles pour que la condition de surface libre puisse être linéarisée et écrite sur le plan horizontal $z=0$. Cette dernière hypothèse a pour conséquence que seul le modèle de houle d'amplitude infinitésimale d'Airy peut être retenu.

\subsubsection{Hypothèse particulière à la zone des atténuateurs}

Les amplitudes des oscillations des structures sont supposées être suffisamment faibles pour que la condition de glissement sur leurs parois puisse être écrite sur la position moyenne occupée par celles-ci au cours du temps.

\subsubsection{Hypothèse particulière en dehors de la zone des at ténuateurs}

La profondeur d'eau, notée $h(M)$, est supposée varier lentement par rapport à la longueur d'onde de la houle pour que les termes du second ordre de la condition de glissement sur le fond soient négligeables.

\subsection{Problème aux limites dans le domaine temporel}

Il résulte de ce qui précède que le champ des vitesses absolues $\vec{V}(M ; t)$ dérive d'un potentiel scalaire harmonique $\Phi(M ; t)$.

On note $\mathcal{D}$ le domaine fluide limité par les carènes $\mathcal{C}=\bigcup_{i=1}^{N} \mathcal{C}_{i}$, la surface libre $S L$, la bathymétrie $F$ (fond, plages, digues et quais) et la surface cylindrique d'axe vertical $\Sigma$ qui matérialise la limite du domaine fluide d'étude avec le large. Par convention, $\vec{n}(M ; t)$ désigne la normale intérieure au fluide. 
Le problème consiste alors à déterminer la fonction potentiel des vitesses $\Phi(M ; t)$ qui satisfait le problème aux limites:

$$
\left\{\begin{array}{lll}
\Delta \Phi(M ; t)=0 & \forall M \in \mathcal{D} & (-h(M) \leq z \leq 0) \\
\frac{\partial^{2} \Phi}{\partial t^{2}}(M ; t)+g \frac{\partial \Phi}{\partial z}(M ; t)=0 & \forall M \in S L & (z=0) \\
\frac{\partial \Phi}{\partial n}(M ; t)=\vec{V}_{E i}(M ; t) \cdot \vec{n}(M ; t) & \forall M \in \mathcal{C}_{i}, & \forall i \in[1, N] \\
\frac{\partial \Phi}{\partial n}(M ; t)=0 & \forall M \in F & (z=-h(M))
\end{array}\right.
$$

expression dans laquelle $\vec{V}_{E i}(M ; t)=\vec{C}_{i}(t)+\vec{\Omega}_{i}(t) \wedge \overrightarrow{G_{i} M}$ désigne la vitesse d'entrainement de la structure $i$, en notant respectivement $\vec{C}_{i}(t)$ et $\vec{\Omega}_{i}(t)$ la vitesse absolue du centre de gravité $G_{i}$ de la structure $i$ d'une part, et son vecteur vitesse de rotation instantanée d'autre part.

Par ailleurs, une condition de radiation doit exprimer que les ondes de perturbation ne se réfléchissent pas sur la frontière de raccord $\Sigma$ avec le large.

\subsection{Problème aux limites dans le domaine fréquentiel}

Dans le cadre d'une théorie linéaire, il est possible de passer du domaine temporel au domaine fréquentiel en écrivant chaque grandeur $G$ sous la forme $G(M ; t)=\Re\left\{G(M ; \omega) e^{-i \omega t}\right\}$.

Le potentiel total $\phi(M ; \omega)$ est la somme du potentiel de la houle incidente $\phi_{I}(M ; \omega)$ et du potentiel de perturbation $\phi_{P}(M ; \omega)$ dû̀ la présence des structures, avec :

$$
\phi_{I}(M ; \omega)=-\frac{a g}{\omega} \frac{\operatorname{ch~} k(z+h)}{\operatorname{ch} k h} e^{i k x}
$$

où $k_{\infty}=\omega^{2} / g$ désigne le nombre d'onde en profondeur illimitée et $k$ le nombre d'onde en profondeur finie, relié à $k_{\infty}$ par la relation de dispersion $k$ th $k h=k_{\infty}$. La fonction complexe $\phi_{P}(M ; \omega)$ indépendante du temps doit alors être solution du problème aux limites suivant:

$$
\left\{\begin{array}{lll}
\Delta \phi_{P}(M ; \omega)=0 & \forall M \in \mathcal{D} & (-h(M) \leq z \leq 0) \\
\frac{\partial \phi_{P}}{\partial z}(M ; \omega)-k_{\infty} \phi_{P}(M ; \omega)=0 & \forall M \in S L & (z=0) \\
\frac{\partial \phi_{P}}{\partial n}(M ; \omega)=-\frac{\partial \phi_{I}}{\partial n}(M ; \omega)+\vec{V}_{E i}(M) \cdot \vec{\pi}(M) & \forall M \in \mathcal{C}_{i}, \quad \forall i \in[1, N] \\
\frac{\partial \phi_{P}}{\partial n}(M ; \omega)=0 & \forall M \in F & (z=-h(M)) \\
\text { Condition de radiation } & \forall M \in \Sigma
\end{array}\right.
$$

\subsection{Les problèmes aux limites élémentaires}

Toutes les équations du problème aux limites (3) étant linéaires, il est possible d'en décomposer la solution en une combinaison linéaire de $6 N+1$ problèmes élémentaires définis à partir des $6 N+1$ termes du second membre de la condition de glissement sur les $N$ carènes. La fonction $\phi_{P}\left(M ; \omega^{\prime}\right)$ est alors obtenue comme la somme d'un terme de diffraction et de $6 N$ termes de radiation. 


\section{Méthode de résolution du problème}

\subsection{Stratégie de résolution du problème}

L'étude d'un site côtier conduit nécessairement̀ à modéliser une étendue plane dont les dimensions se mesurent en centaines de mètres, voire en kilomètres. Comme il est nécessaire d'avoir au moins cinq ou six éléments de discrétisation par longueur d'onde de la houle, ceux-ci doivent avoir au plus des dimensions de l'ordre de quelques mètres. Il en résulte que le nombre d'éléments de la discrétisation peut aller de quelques milliers pour les sites peu étendus à quelques dizaines de milliers pour les sites les plus importants.

L'utilisation d'un modèle entièrement tridimensionnel n'apparaît donc pas être des plus rationnelles, en particulier pour la modélisation de sites à la bathymétrie complexe pour lesquels l'utilisation de fonctions de Green est plus délicate. La solution la plus performante et la plus économique, pour de telles étendues, est de recourir à l'utilisation d'un modèle fondé sur la formulation bidimensionnelle en plan proposée en 1972 par J.C.W. Berkhoff [1] et mise en cuvre dans un programme d'éléments finis linéaires et bilinéaires.

Il n'est toutefois pas possible, dans ce type de modèle, de tenir compte d'objets ou d'obstacles tridimensionnels induisant des vitesses verticales importantes. Il convient donc d'opter autour des atténuateurs pour l'utilisation locale du modèle tridimensionnel de diffraction-radiation et pour l'utilisation du modèle de Berkhoff en dehors de cette zone. Les difficultés liées au couplage des deux méthodes sont alors largement compensées par les performances obtenues.

\subsection{Résolution en dehors de la zone des atténuateurs}

\subsubsection{Equation indéfinies}

La recherche d'une solution à variables séparée permet de dissocier le problème vertical du problème horizontal. La pente des fonds étant relativement faible le potentiel des vitesses peut se mettre sous la forme:

$$
\phi(x, y, z ; \omega)=-\frac{i g}{2 \omega} \psi(z) H(x, y ; \omega)=-\frac{i g}{2 \omega} \frac{\operatorname{ch~} k(z+h)}{\operatorname{ch~} k h} H(x, y ; \omega)
$$

où la fonction complexe $H(x, y ; \omega)$ représente le double de la dénivelée de la surface libre. $\phi(x, y, z ; \omega)$ devant vérifier l'équation de Laplace tridimensionnelle, $H(x, y ; \omega)$ doit vérifier l'équation d'Helmholtz bidimensionnelle: $\Delta_{2} H+k^{2} H=0$. La solution au premier ordre ne donnant pas la répartition spatiale de $H(x, y ; \omega)$, le deuxième ordre est pris en compte en appliquant la deuxième formule de Green, par rapport à la variable $z$ sur le support $[-h, 0]$, aux deux fonctions $\psi$ et $\phi$. La condition aux limites sur le fond se traduit alors par une condition sur les seules variations horizontales représentées par le gradient et le laplacien bidimensionnels de $\phi$ dont seuls les termes du premier ordre seront conservés.

D'où l'équation de Berkhoff qui doit être vérifiée en tout point du plan d'eau :

$$
\operatorname{div}_{2}\left(C C_{g} \overrightarrow{\operatorname{grad}}_{2} H\right)+\omega^{2} \frac{C_{g}}{C}\left(1+i \nu_{t}\right) H=0
$$

Expression dans laquelle $C_{g}$ désigne la vitesse de groupe et $\nu_{t}$ caractérise le frottement tangentiel sur le fond obtenu à partir de la condition de surface 
libre du fluide presque parfait, ce qui correspond à l'introduction d'un effort de dissipation par unité de masse $-2 \varepsilon \vec{V}$ dans l'équation d'Euler, avec:

$$
\nu_{t}=\frac{4 \varepsilon}{\omega} \frac{\operatorname{sh} 2 k h}{2 k h+\operatorname{sh} 2 k h}
$$

\subsubsection{Conditions aux limites}

L'équation de Berkhoff doit être satisfaite dans un domaine $\Omega(x, y)$ du plan horizontal limité par une frontière $\Gamma(x, y)$. Les conditions aux limites qui doivent être satisfaites sur la frontière $\Gamma$ peuvent être classées en trois catégories correspondant aux trois types de frontières:

- les frontières terrestres $\Gamma_{T}$ (quais, digues, plages, murs poreux ...) sur lesquelles sera écrite une condition de réflexion partielle avec urie incidence généralement normale à la paroi (coefficient $R$ de réflexion normale et angle $\theta$ nul) sous la forme :

$$
\frac{\partial \phi}{\partial n}-i k \cos \theta \frac{1-R}{1+R} \phi=0
$$

- les frontières de raccord $\Gamma_{E}$ avec le milieu infini extérieur $\Omega_{E}$ (projection horizontale de $\Sigma$ ) qui est soumis à l'action de la houle. Le domaine extérieur est semi-infini. Sa géométrie est supposé être simple. La profondeur h est constante. Il est délimité par deux lignes semi-infinies représentant le trait de côte de part et d'autre du site côtier, reliées entre elles par un arc de cercle. Dans le domaine semi-infini, le potentiel extérieur est la somme des potentiels de l'onde incidente du large, des ondes de réfléxion de l'onde incidente sur les parois extérieures au port et de l'onde radiée par le port. Ce dernier est décomposé sur une base de fonctions propres solutions du problème extérieur. Il en résulte que les conditions sur la frontière $\Gamma_{E}$ se traduisent par une matrice d'impédance complexe pleine (pour ses éléments) qui affecte le premier membre du système linéaire et un vecteur d'excitation qui en affecte le second membre. - Les frontières de raccord $\Gamma_{I}$ avec un domaine de calcul intérieur par une méthode différente $\Omega_{I}$, sur lesquelles il convient d'assurer la continuité de la fonction potentiel des vitesses et de sa dérivée normale. A l'instar de ce qui a été fait dans le cas du milieu infini extérieur, le couplage entre les deux méthodes s'effectue formellement en introduisant dans les conditions aux limites du domaine $\Omega$ sur la frontière $\Gamma_{I}$ une matrice d'impédance $\left[Z_{I}\right]$ qui permet d'exprimer le vecteur correspondant à la dérivée normale de $\phi$ en fonction du vecteur correspondant à $\phi$ :

$$
\left[\frac{\partial \phi}{\partial n}\right]=\left[Z_{I}\right][\dot{\phi}]
$$

\subsection{Résolution dans la zone des atténuateurs}

\subsubsection{Discrétisation de la zone des attémuateurs}

La modélisation de la zone des atténuateurs est réalisée à partir d'une formulation tridimensionnelle par équations intégrales mettant en œuvre, pour sa résolution, une méthode de singularités mixtes de Green. La surface libre, les 
atténuateurs, le fond, et les éventuels quais sont discrétisés en facettes. Les conditions aux limites y sont imposées numériquement. La discrétisation du contour de la zone des atténuateurs est imposée par le maillage d'éléments finis du domaine $\Omega$. Chacun des $N_{I}$ segments de $\Gamma_{I}$ qui est commun aux deux maillages, engendre, pour la modélisation tridimensionnelle, une facette trapézoïdale verticale délimitée par la surface libre, le fond et deux segments verticaux.

\subsubsection{Décomposition du problème hvdrodvnamique}

La colonne $i$ de la matrice d'impédance est construite à partir de la solution du problème "d'excitation" obtenu, les atténuateurs étant immobiles, en imposant sur la facette $i$ de la frontière $\Gamma_{I}$ un potentiel correspondant à la fonction :

$$
\psi(z) \cos \omega t=\frac{\operatorname{ch} k(z+h)}{\operatorname{ch} k h} \cos \omega t
$$

et un potentiel nul sur les autres facettes de la frontière.

Il est évident que cette excitation élémentaire engendre sur les différents atténuateurs des efforts d'excitation auquels ceux-ci répondent en s'animant d'un mouvement sinusoïdal. Il faut donc à chaque fois résoudre l'équation de la mécanique des atténuateurs pour connaitre leurs mouvements suivant leurs six degrés de liberté respectifs. Pour celà, les coefficients hydrodynamiques des atténuateurs sont préalablement déterminés en imposant successivement à chaque degré de liberté des atténuateurs une vitesse unitaire, les autres étant bloqués, et un potentiel nul partout sur les facettes de la frontière $\Gamma_{I}$; ce qui revient à résoudre $6 \mathrm{~N}$ problèmes de "radiation".

Les termes de la $i$ ème colonne de la matrice d'impédance sont obtenus comme des combinaisons linéaires des vitesses normales qui résultent, sur les différents éléments de la frontière, du problème d'excitation et des problèmes de radiation. Il convient de noter qu'en abscence de sources de dissipation d'énergie, la matrice d'impéclance est réelle, et que l'adjonction d'une source d'énergie dans le domaine tridimensionnel (batteur à houle par exemple) engendrerait un vecteur d'excitation qui affecterait également le second membre du système.

\subsubsection{Comportement des structures}

Le comportement de chaque atténuateur est déterminé en lui appliquant l'équation de Newton, qui stipule l'égalité à chaque instant entre le torseur de ses efforts d'inertie et celui des efforts extérieurs qu'il subit. Cette équation est résolue au centre de gravité de chaque module d'atténuateur.

Les efforts extérieurs appliqués à chaque module sont:

- les efforts dus à la pression du fluide qui se composent des éléments suivants :

- les efforts hydrodynamiques d'inertie en phase avec l'accélération (masses et inerties d'eau ajoutées dans la boite de contrôle),

- les efforts hydrodynamiques d'excitation indépendants des mouvements (efforts engendrés sur la structure supposée fixe par les potentiels d'excitation incidents et diffractés dus aux "impulsions"),

- les efforts hydrostatiques en phase avec la position.

- les efforts dus à la gravité (poids) qui sont combinés aux efforts hydrostatiques pour obtenir les rappels hydrostatiques par rapport à l'équilibre statique. 
- les efforts dus aux liaisons extérieures (amarres et ancrages).

- des efforts d'amortissement visqueux qui sont éventuellement introduits au moyen d'une formulation de type Morison linéarisée appropriée.

\section{Utilisation de résultats bidimensionnels}

Dans certains cas, il peut être intéressant d'utiliser des résultats expérimentaux pour la modélisation des atténuateurs. Si les essais ont été conduits en tridimensionnel (cuve à houle), il est possible, sous réserve que le nombre et la disposition des sondes de houle soient adéquats, d'en déduire directement une matrice d'impédance tridimensionnelle complexe.

Toutefois ce sont, le plus souvent, des résultats expérimentaux bidimensionnels qui sont disponibles (calculs ou essais en canal à houle), et il est toujours intéressant de pouvoir les utiliser pour modéliser une ligne d'atténuateurs sur un site côtier. En désignant respectivement par $R(\omega)$ et $T(\omega)$ les coefficients de réflexion et de transmission complexes (modules et phases), les amplitudes complexes amont $\left(H^{-}(x, y ; \omega)\right)$ et aval $\left(H^{+}(x, y ; \omega)\right)$ vérifient, le long de la ligne sans épaisseur représentant les atténuateurs, la relation :

$$
\left[\begin{array}{l}
\frac{\partial}{\partial n} H^{-} \\
\frac{\partial}{\partial n} H^{+}
\end{array}\right]=-\frac{i k}{(1+R)^{2}-T^{2}}\left[\begin{array}{cc}
1-R^{2}+T^{2} & -2 T \\
-2 T & 1-R^{2}+T^{2}
\end{array}\right]\left[\begin{array}{l}
H^{-} \\
H^{+}
\end{array}\right]
$$

Ces matrices complexes symétriques sont calculées à partir des résultats expérimentaux (modules et phases) pour chaque période et chaque profondeur. La matrice d'impédance globale est alors diagonale par blocs de dimension 2 .

Une fois le problème résolu, les fonctions $H^{-}(x, y ; \omega)$ et $H^{+}(x, y ; \omega)$ sont connues et il est possible de déterminer les caractéristiques des "houles incidentes" correspondantes. Les efforts normaux à la ligne d'atténuateurs qui en résultent peuvent être calculés à leur tour au moyen des résultats bidimensionnels. Cette méthode, qui présente un nireau d'approximation, lié au passage du tridimensionnel en bidimensionnel, analogue à celui qui résulte de l'utilisation des coefficients de réflexion partielle sur les frontières terrestres, présente l'intérêt de la. simplicité et de l'efficacité, surtout lorsque la ligne d'atténuateurs est longue.

\section{Application au cas de Dieppe}

Le poste d'amarrage des car-ferries de Dieppe ayant été transféré de l'avantport (zone A) dans le port extérieur plus vaste (zone B), les autorités locales ont décidé d'installer un port de plaisance dans la darse de l'avant-port rendue ainsi disponible. Toutefois, la hauteur crête à creux de la houle résiduelle dans cette darse étant de l'ordre d'un mètre dix dans les conditions annuelles, et d'un mètre cinquante dans les conditions trentenales, cette nouvelle affectation des lieux rendait nécessaire l'installation d'une protection anti-houles. Pour des raisons techniques et économiques, seule la solution fondée sur l'utilisation d'atténuateurs flottants ASB-5000 pouvait raisonnablement être envisagée.

Le modèle numérique couplé a donc été mis en œuvre pour prévoir l'évolution de l'agitation de la darse en fonction du nombre des modules atténuateurs et 
de leur disposition. Il a été validé (amortissements sur le fond, coefficients de réflexion partielle) par comparaison avec les résultats obtenus, sans atténuateur, au réel et sur le modèle physique au 1/71 ème réalisé par la Sogreah.

Ensuite les atténuateurs ont été pris en compte (zone C), d'abord au moyen du modèle numérique tridimensionnel validé par rapport aux résultats numériques et expérimentaux connus en diffraction-radiation, puis au moyen du modèle expérimental réalisé au $1 / 15$ et au $1 / 20$ en canal à houle à EDF à Chatou [2]. Le modèle numérique comprend 13233 nœuds pour la zone éléments finis et 1326 facettes pour la zone des singularités. Grâce à la performance du modèle hybride, les calculs d'agitation ont pu être réalisés sur un micro-ordinateur $486 \mathrm{DX} 266 \mathrm{MHz}$ en $75 \mathrm{mn}$ par période (de $4 \mathrm{~s}$ à 15s) sans atténuateurs et en $90 \mathrm{mn}$ avec atténuateurs, pour trois incidences. La matrice d'impédance nécessitant $45 \mathrm{mn}$ de calcul par période.

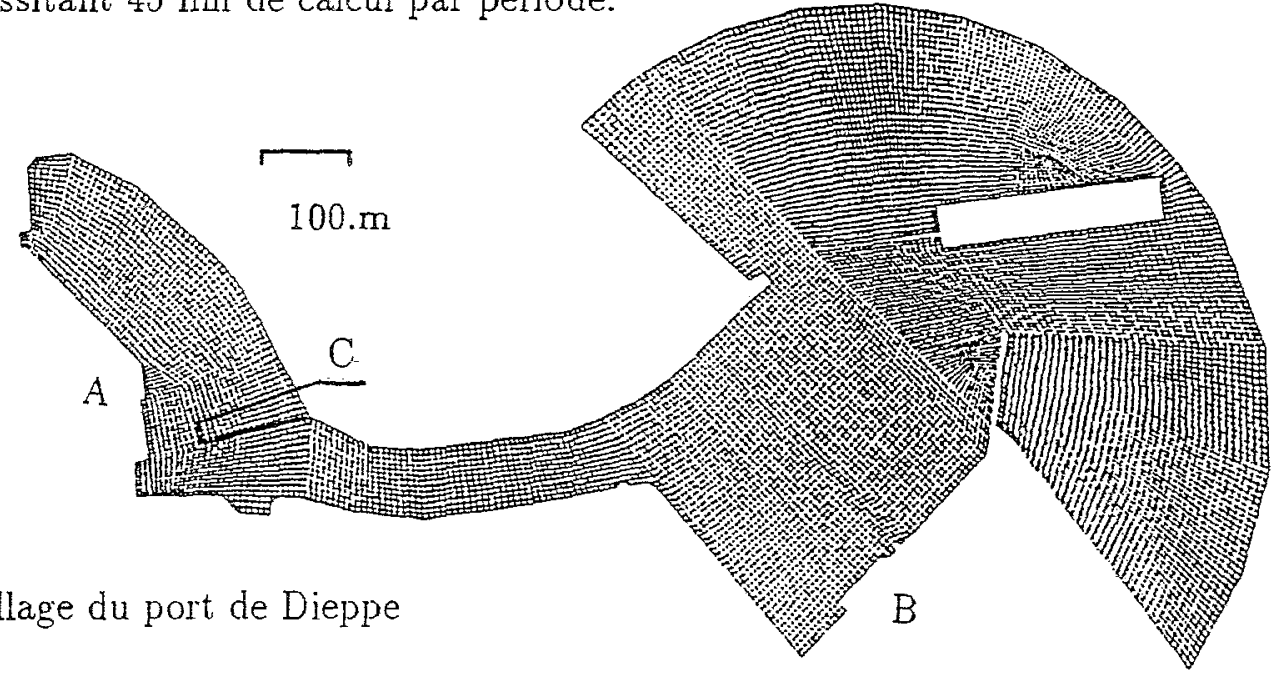

\section{Conclusion}

Le projet d'implantation d'atténuateurs de houles ASB-5000 pour protéger la darse de plaisance de l'avant-port de Dieppe nous a conduit à développer et à valider un modèle d'agitation portuaire bidimensionnel plan capable de prendre en compte localement un ensemble de structures mobiles indéformables tridimensionnelles. Ce modèle, développé de manière générale, nous permet désormais de prévoir, dans des conditions économiques raisonnables, l'impact des aménagements proposés lors des avant-projets et projets de protection des plans d'eau par des structures fixes ou flottantes tridimensionnelles.

Naturellement, cet outil numérique est également adapté à l'étude du comportement hydronécanique des navires dans des ports ou dans des eaux confinées.

\section{Références}

[1] J.C.W. Berkhoff, "Computation of combined refraction-ziffraction" - Proc. 13th Coastal Engineering Conference pp 471-490 - ASCE 1972.

[2] J. Bougis et A. Degaie, "L'ASB-5000: un nouveau concept d'atténuateurs de houles" - Cités Marines'95 - Monaco Novembre 1995. 